\title{
Supporting Information: Modeling the Ultrafast Electron Attachment Dynamics of Solvated Uracil
}

Cate S. Anstöter, ${ }^{*, \dagger}$ Mark DelloStritto, ${ }^{*, \ddagger}$ Michael L. Klein, ${ }^{,, \ddagger}$ and Spiridoula Matsika ${ }^{*} \dagger$

$\dagger$ Department of Chemistry, Temple University, Philadelphia, PA 19122, USA

$\ddagger$ Institute for Computational Molecular Science, Temple University SERC, Philadelphia, PA 19122, USA

E-mail: csanstoter@gmail.com; tuh39227@temple.edu; mlklein@temple.edu; smatsika@temple.edu 


\section{Uracil Solvation}

We investigated the impact of finite size effects on the solvation of uracil using the GAFF potential. ${ }^{1}$ We simulated uracil solvated by $32,64,128$, and $256 \mathrm{H}_{2} \mathrm{O}$ molecules using the $\mathrm{GAFF}^{1}$ and computed the radial distribution functions (RDFs) of the atoms of the uracil molecule with reference to the $\mathrm{O}$ atoms of the $\mathrm{H}_{2} \mathrm{O}$ molecules. We plot the RDFs in Figures S1, S2, and S3 where it should be apparent that the difference in the solvation structure between 128 and 256 molecules is relatively small. While there are small changes in the RDFs when increasing from 128 to $256 \mathrm{H}_{2} \mathrm{O}$ molecules, the differenc is small compared to increasing from 64 to 128 molecules and is mostly confined to larger distances. Thus, simulating uracil in water with $128 \mathrm{H}_{2} \mathrm{O}$ molecules is a good compromise between accuracy and efficiency for ab-initio simulations.

We also observed that the uracil anion exhibits much stronger H-bonds with water compared to neutral uracil. In particular, we saw that the $\mathrm{H}$ atoms of water show a strong attraction to the $\mathrm{C}$ atoms of the uracil anion, as evidenced by the shift in the $\mathrm{C}-\mathrm{Hw} \mathrm{RDF}$ in Figure S4. This is likely due to the significant negative partial charge of the $\mathrm{C}$ atoms of the uracil anion, such that the water molecules are forming what may be thought of as H-bonds with the pi-attached excess electron in uracil.

We list in Table S1 the iterative Hirshfeld charges of each atom of the uracil anion averaged over each simulation at the final timestep, with the labels associated with each atom shown in Figure S7. 


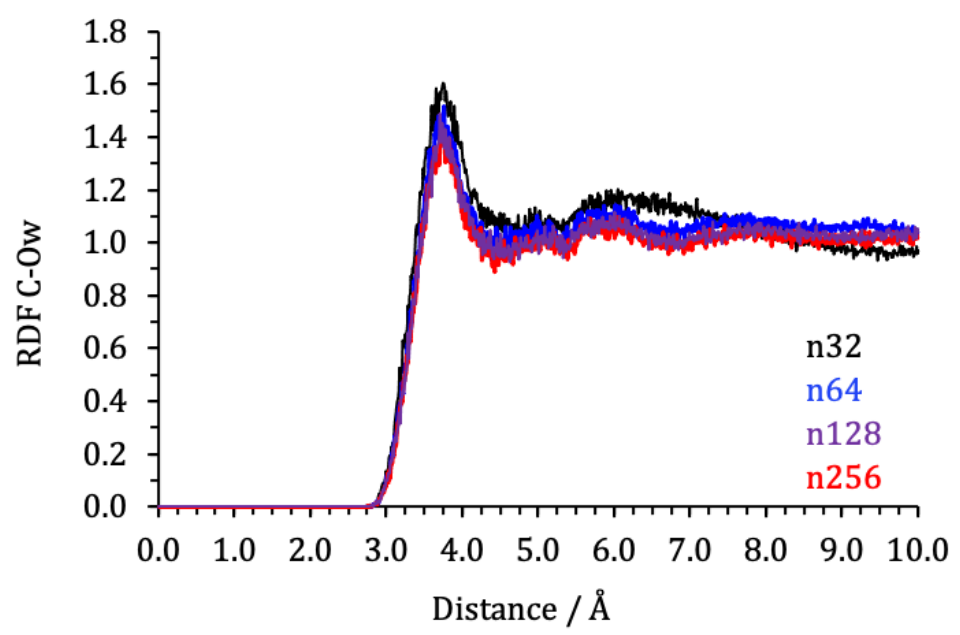

Figure S1: Radial distribution function for the $\mathrm{C}$ atoms of neutral uracil to the $\mathrm{O}$ atoms of water $(\mathrm{Ow})$ for uracil solvated by $32,64,128$, and $256 \mathrm{H}_{2} \mathrm{O}$ molecules using a classical force field (GAFF).

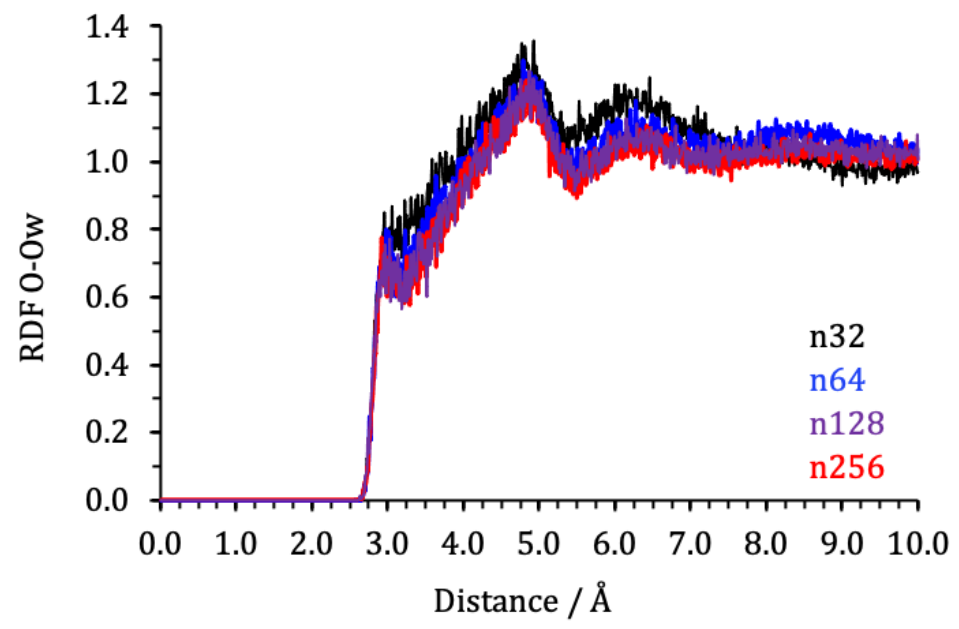

Figure $\mathrm{S} 2$ : Radial distribution function for the $\mathrm{O}$ atoms of neutral uracil to the $\mathrm{O}$ atoms of water $(\mathrm{Ow})$ for uracil solvated by $32,64,128$, and $256 \mathrm{H}_{2} \mathrm{O}$ molecules using a classical force field (GAFF). 


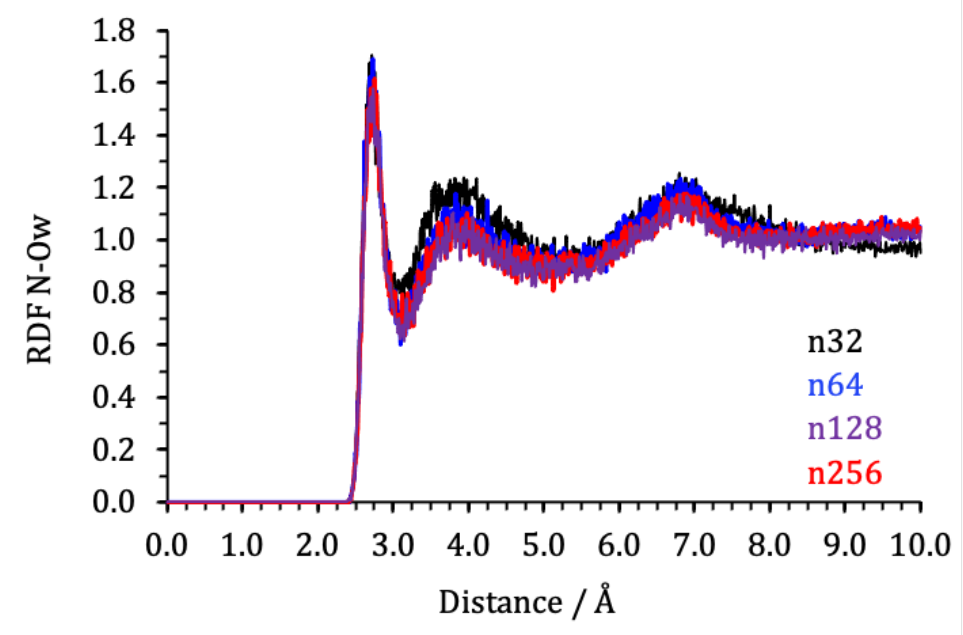

Figure S3: Radial distribution function for the $\mathrm{N}$ atoms of neutral uracil to the $\mathrm{O}$ atoms of water $(\mathrm{Ow})$ for uracil solvated by $32,64,128$, and $256 \mathrm{H}_{2} \mathrm{O}$ molecules using a classical force field (GAFF).

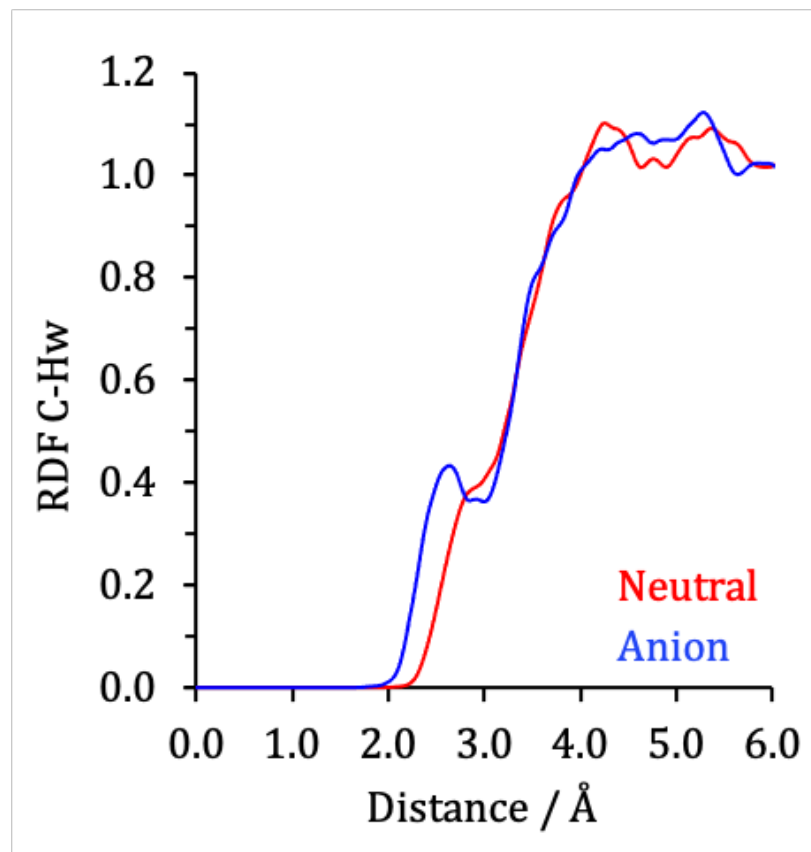

Figure S4: Radial distribution function for the $\mathrm{C}$ atoms of uracil to the $\mathrm{H}$ atoms of water $(\mathrm{Hw})$ for both neutral uracil and the uracil anion averaged over all ab-initio simulations. 


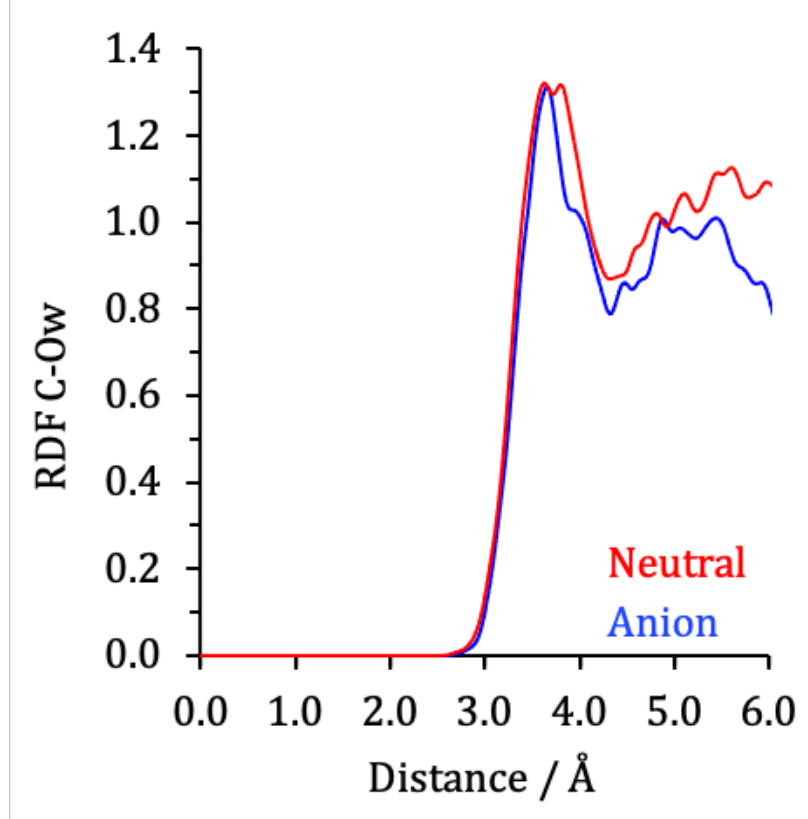

Figure S5: Radial distribution function for the $\mathrm{C}$ atoms of uracil to the $\mathrm{O}$ atoms of water $(\mathrm{Ow})$ for both neutral uracil and the uracil anion averaged over all ab-initio simulations.

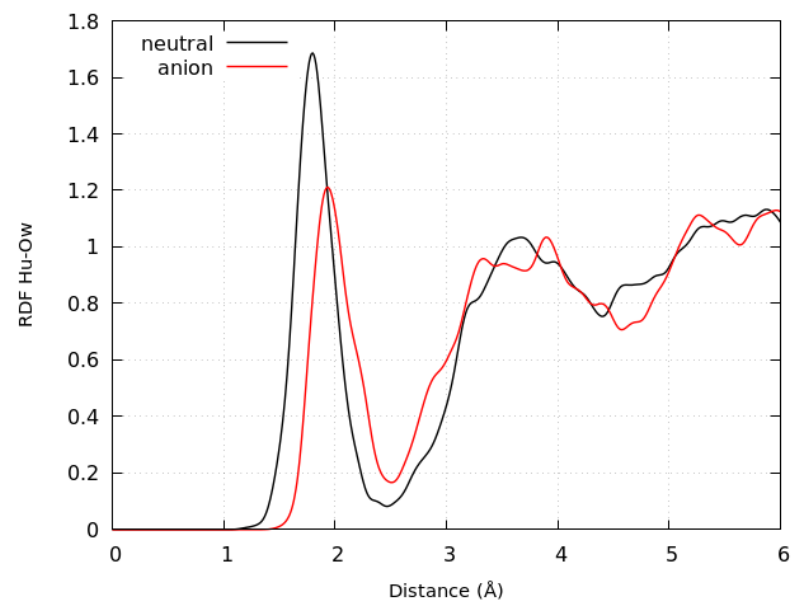

Figure S6: Radial distribution function for the $\mathrm{H}$ atoms of uracil to the $\mathrm{O}$ atoms of water $(\mathrm{Ow})$ for both neutral uracil and the uracil anion averaged over all ab-initio simulations. 
Table S1: Net Iterative Hirshfeld charges for the uracil anion averaged over each simulation at the final timestep.

\begin{tabular}{|r|r|}
\hline N1 & -0.885 \\
\hline N2 & -0.8022 \\
\hline C1 & 0.917 \\
\hline C2 & 0.5732 \\
\hline C3 & -0.447 \\
\hline C4 & -0.7118 \\
\hline O1 & -0.935 \\
\hline O2 & -0.9884 \\
\hline H1 & 0.6702 \\
\hline H2 & 0.6592 \\
\hline H3 & 0.5494 \\
\hline H4 & 0.55 \\
\hline
\end{tabular}

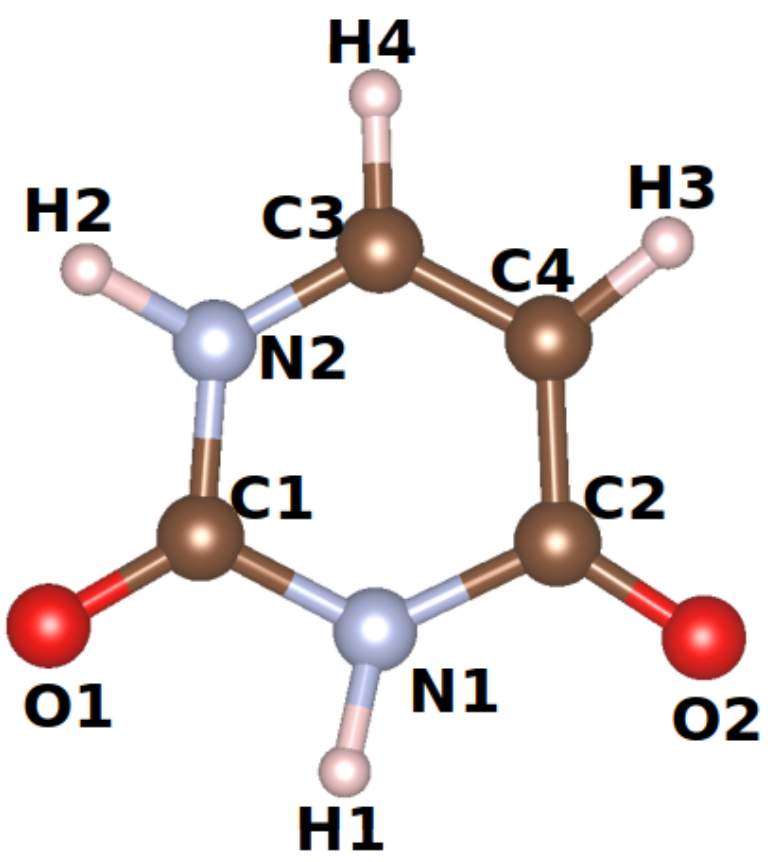

Figure S7: Uracil anion with the labels present in Table S1 added next to each of the atoms. 


\section{Time Dependent Dynamics}
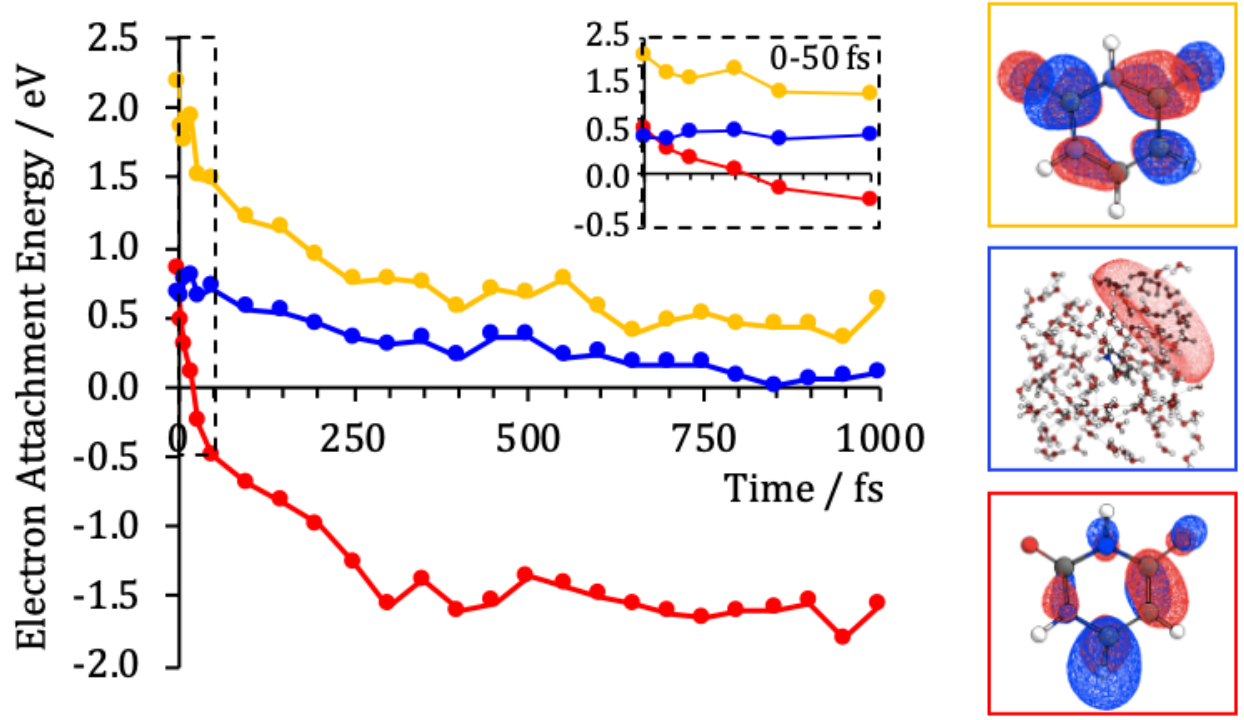

Figure S8: Relative energies for the valence and non-valence electron attachment states of the vibrationally hot uracil core, as a function of simulation time. $t=0$ represents the starting point of the neutral solvated uracil cell, and $t=1$ ps represents the equilibrated charged solvated uracil cell. At early times, the vertical electron attachment energies were calculated at $0,5,10,25,30$ and $50 \mathrm{fs}$, to capture the rapid relaxation dynamics of the charged trajectory, amplified and shown inset. After $50 \mathrm{fs}$, the vertical electron attachment energies were calculated every $\sim 50$ fs. The energies were calculated individually for all of the MD simulations and averaged. The color scheme follows that of the main text. Representative molecular orbitals denoting three electron attachment states are shown to the right of the plot, following the same colour scheme. 
(a)

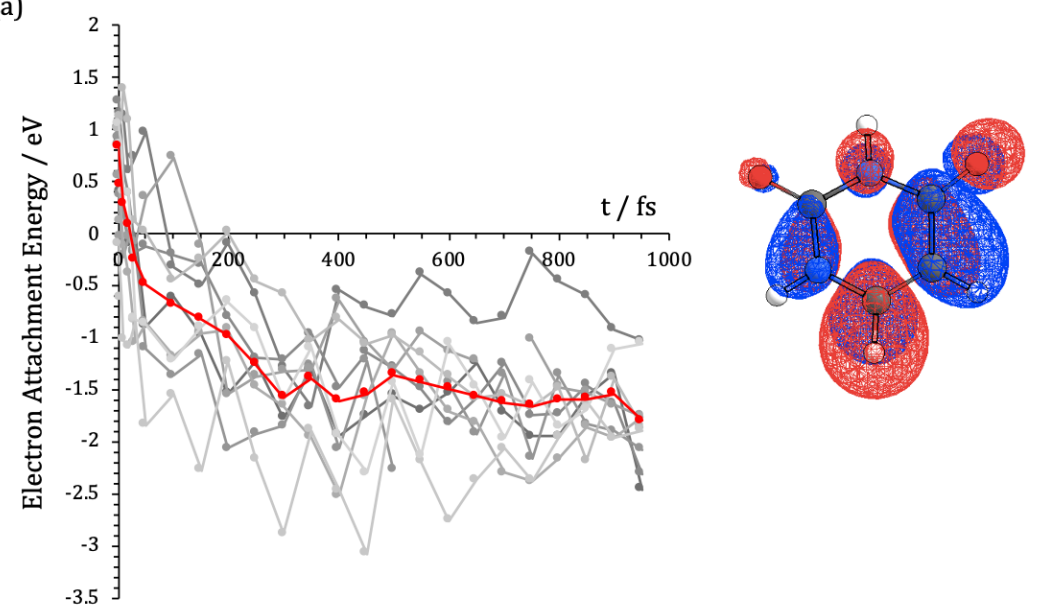

(b)
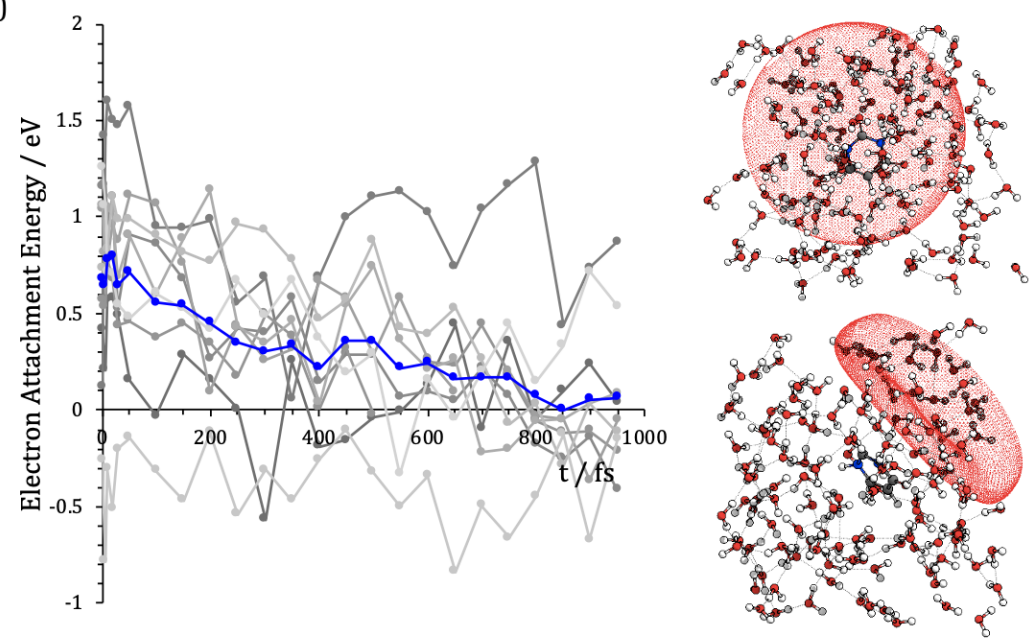

(c)
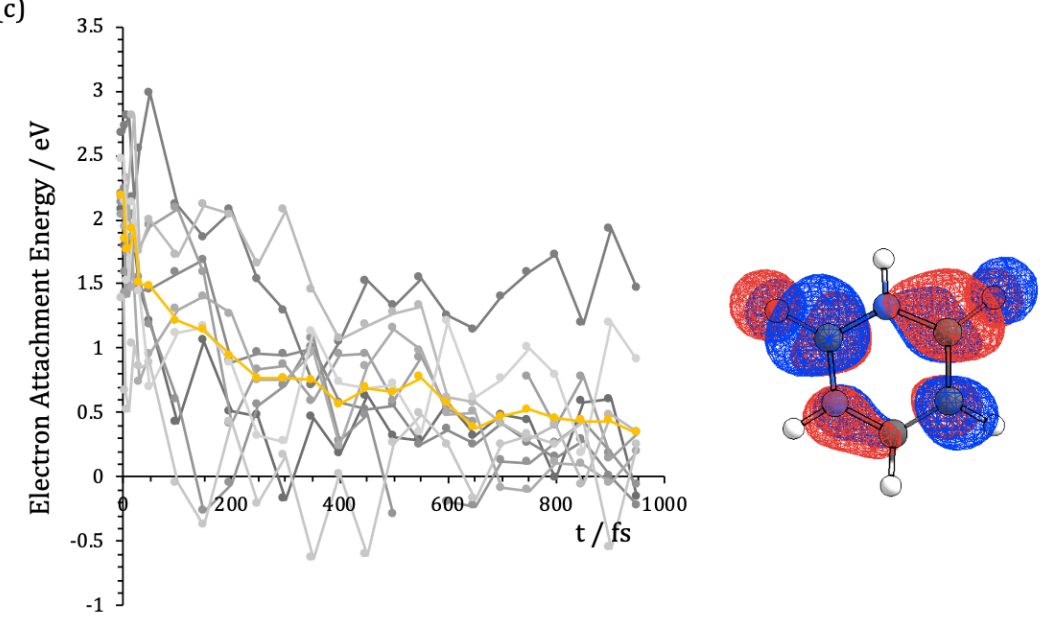

Figure S9: Vertical electron attachment energies calculated for (a) the $\pi_{1}^{*}$, (b) presolvated electron and (c) the $\pi_{2}^{*}$ states plotted as a function of trajectory time for all nine 1 ps neutral to charged trajectories. The individual trajectories are shown in greyscale with the respective average energy for the states following the same colour scheme as the figure in the main text. Representative molecular orbitals are shown to the right. 
(a)

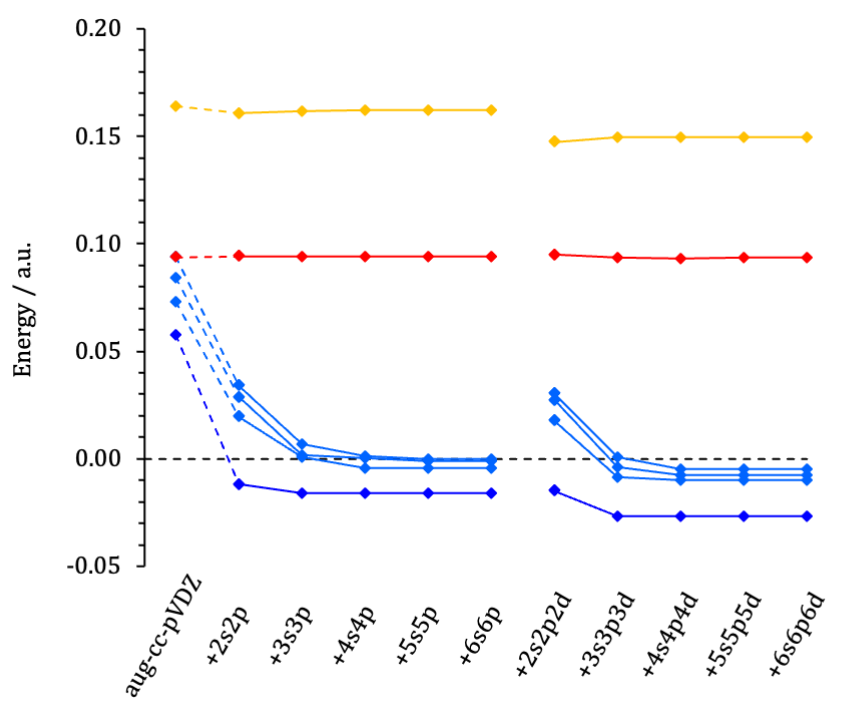

(b)

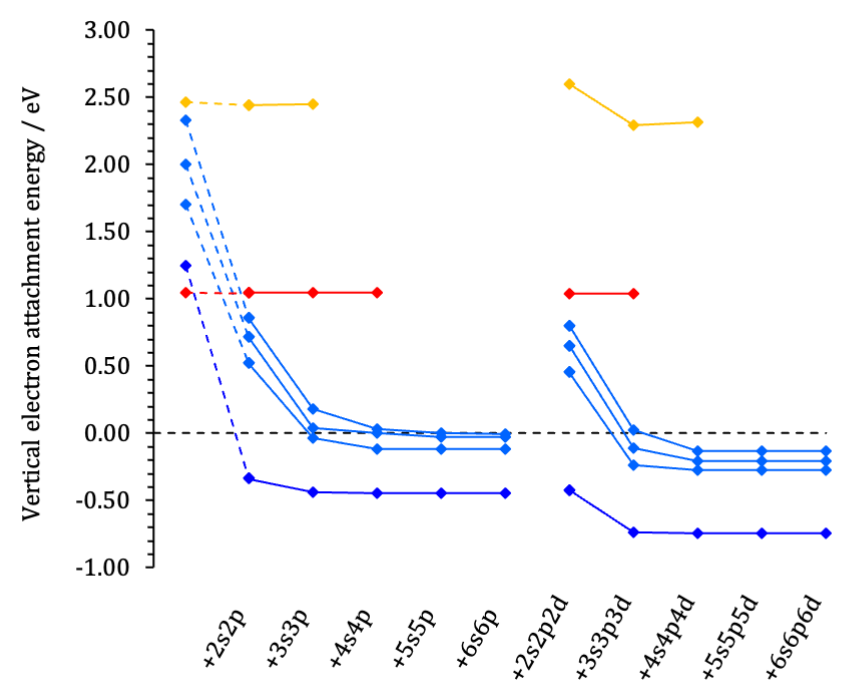

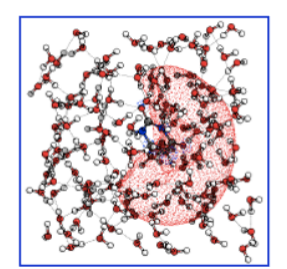
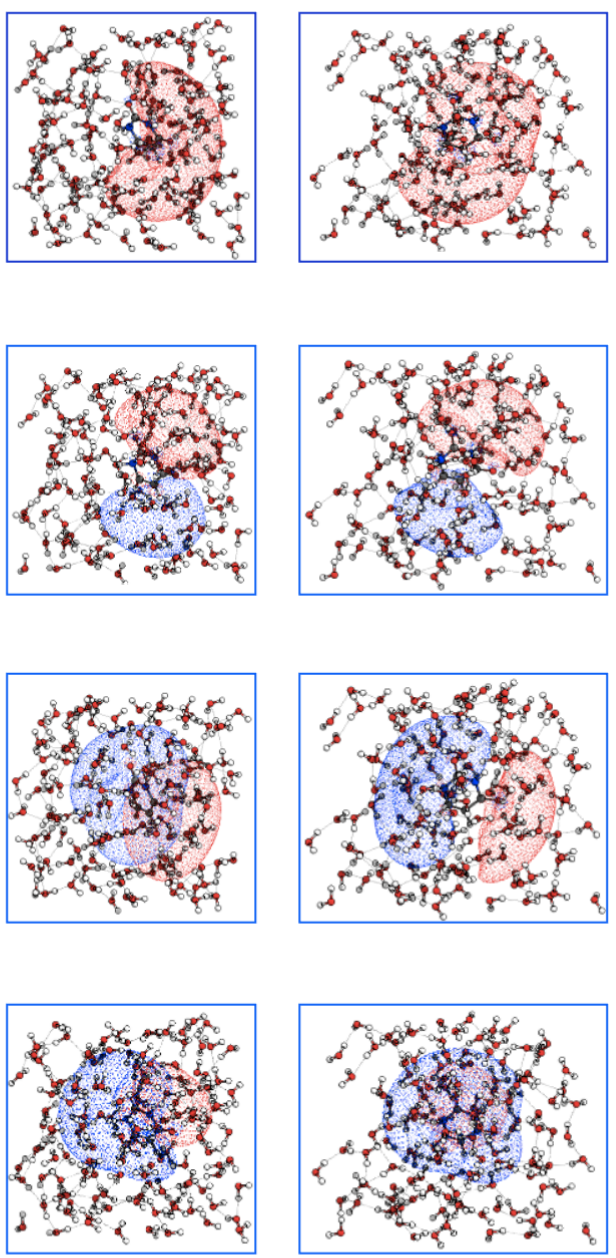

Figure S10: Variation of (a) orbital energy and (b) vertical electron attachment energy with changing $+\mathrm{XsXp}_{\mathrm{X}}(\mathrm{Xd})$ functions affixed to the central ghost atom. For reference the energies for the parent basis set, aug-cc-pVDZ, is included. The valence $\pi_{1}^{*}$ and $\pi_{2}^{*}$ states (red and yellow, respectively) are included alongside solutions of the presolvated electron states with s- and p-symmetry (dark blue and blue, respectively). Representative orbitals for the nonvalence presolvated electron orbitals are shown on to the right, following the color scheme. 
(a)

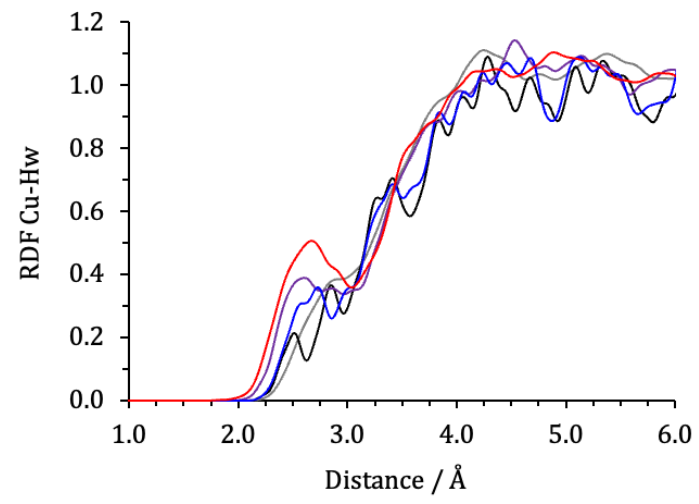

(b)

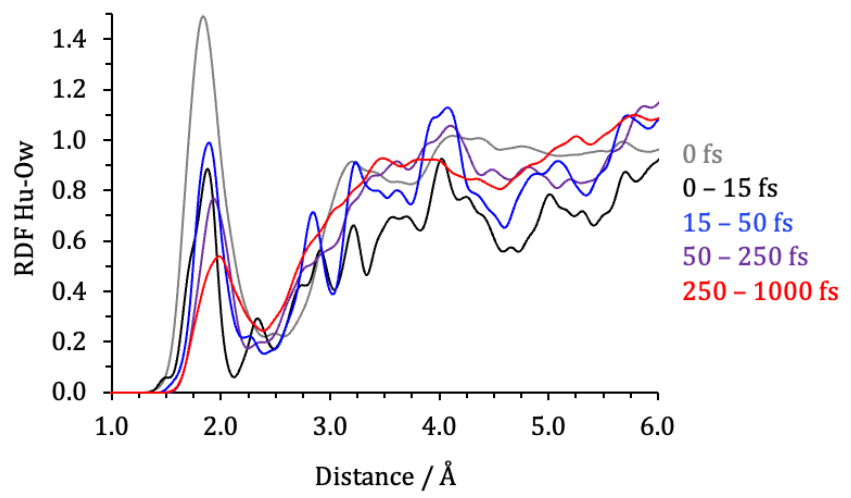

Figure S11: Radial distribution functions for the carbon atoms of uracil and the hydrogen atoms of water (a) and the hydrogen atoms of water and the oxygen atoms of water (b), respectively, shown as a function of time region, fs. The color scheme used for both (a) and (b) is given to the right of the figure.

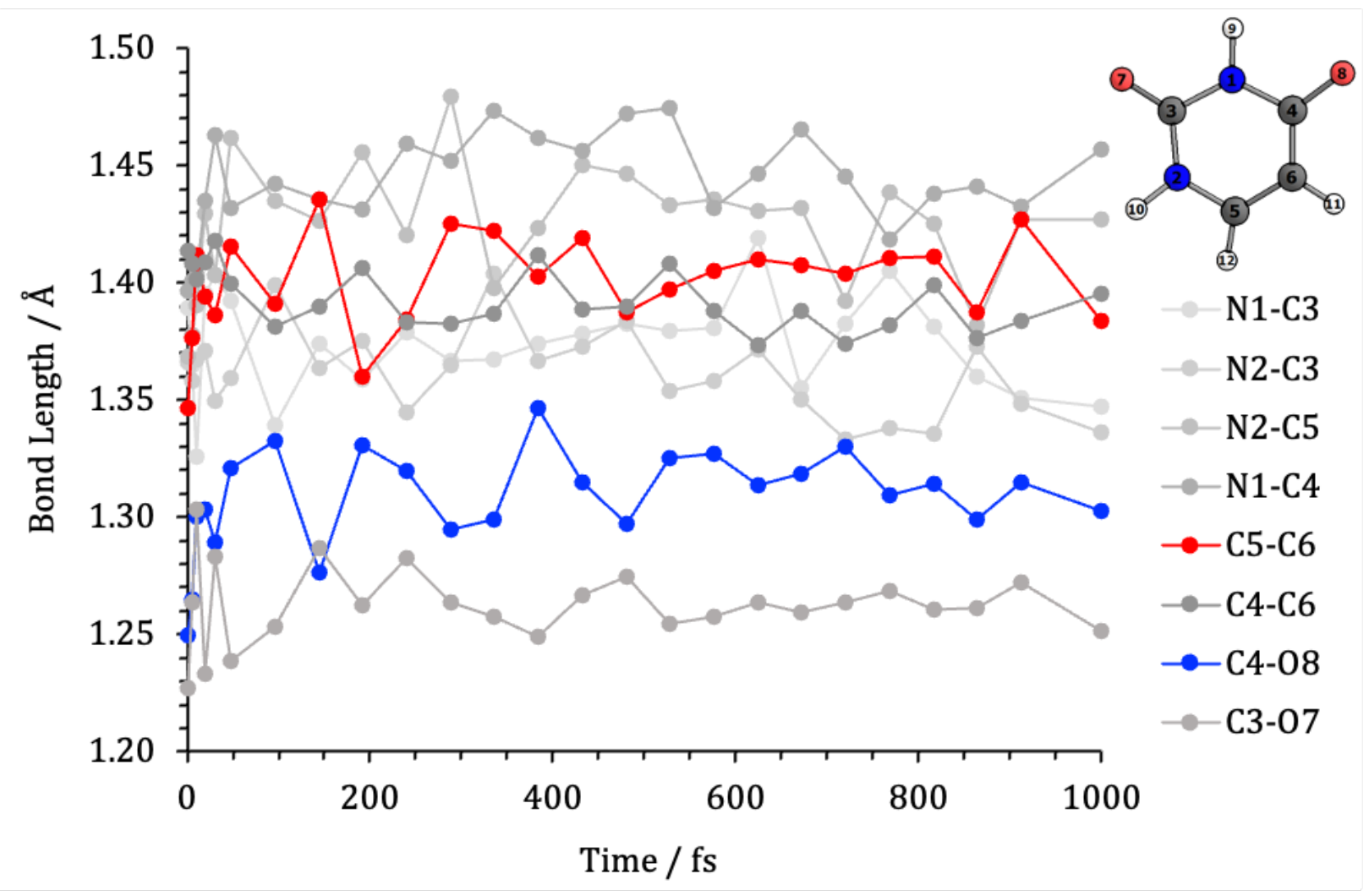

Figure S12: Variation in bond length, $\AA$, averaged for the 9 different SCAN trajectories as a function of simulation time, fs. The numbering system for atoms is included inset, alongside the legend. The $\mathrm{C} 5-\mathrm{C} 6$ and $\mathrm{C} 4-\mathrm{O} 8$ bonds are highlighted as red and blue, respectively. 


\begin{tabular}{|c|c|c|c|c|c|c|c|c|}
\cline { 2 - 9 } \multicolumn{1}{c|}{} & \multicolumn{9}{c|}{ Bond Length / $\AA$} \\
\hline$t$ region / fs & N1-C3 & N2-C3 & N2-C5 & N1-C4 & C5-C6 & C4-C6 & C4-08 & C3-07 \\
\hline 0 & 1.389 & 1.366 & 1.368 & 1.396 & 1.347 & 1.414 & 1.249 & 1.227 \\
$0-10$ & 1.360 & 1.364 & 1.379 & 1.400 & 1.378 & 1.408 & 1.272 & 1.265 \\
$10-50$ & 1.379 & 1.362 & 1.421 & 1.433 & 1.402 & 1.407 & 1.303 & 1.265 \\
$50-250$ & 1.369 & 1.368 & 1.440 & 1.440 & 1.397 & 1.392 & 1.316 & 1.265 \\
$250-1000$ & 1.375 & 1.358 & 1.428 & 1.452 & 1.405 & 1.388 & 1.314 & 1.263 \\
\hline Neutral eqm & 1.389 & 1.395 & 1.378 & 1.412 & 1.361 & 1.466 & 1.223 & 1.219 \\
\hline Anion eqm & 1.38 & 1.371 & 1.44 & 1.443 & 1.432 & 1.414 & 1.262 & 1.253 \\
\hline
\end{tabular}

Figure S13: Averaged changing bond lengths, $\AA$, for all bonds around the uracil ring as a function of the important time regions identified. For comparison, the corresponding bond lengths of the optimized planar neutral and the bent anion geometries are given below. We note that the bond lengths for the identified time regions are taken from the nine molecular dynamics trajectories obtained using SCAN while the equilibrium geometries were optimized using MP2, for this reason absolute agreement is not expected. The comparison is simply to illustrate the expected elongation or contraction of bond lengths. 


\section{Cartesian Coordinates}

Neutral equilibrium geometry, $\left(\mathrm{S}_{0 e q m}\right)$

N -0.801933052 -0.240108122 -1.009637838

N $1.1422660980 .716896591-0.195546563$

C $0.3339535300 .499967078-1.311999075$

C -1.216029195 -0.769420569 0.232675675

C 0.8454803810 .2505764191 .066330434

C -0.280435780 -0.470585539 1.320539884

O $0.6075251410 .919358187-2.423863500$

O -2.258450595 - 1.4024322290 .327048287

H -1.421386400 -0.421542280 - 1.798618902

H $1.9845626871 .254735694-0.372115516$

H -0.508726216 -0.836441287 2.321492031

H 1.5731734020 .4989960571 .843695082

Anion equilibrium geometry, $\left(\mathrm{D}_{0 e q m}\right)$

N -0.801659406 -0.233256243 -1.006833061

N $1.1973744630 .645126323-0.232467298$

C $0.3488684710 .476826964-1.292623721$

C -1.204783413 -0.7802881460 .276826818$

C 0.8964104430 .2420071131 .105737310

C -0.321389043 -0.456083127 1.329280421

O $0.5992694190 .909493767-2.441879179$

O $-2.272529168-1.4435429680 .284432067$

H -1.429551364 -0.353657718 - 1.789669975

H $1.9442505961 .300705693-0.423582757$

H -0.554030177 -0.8629301342.316185642

H 1.5977691810 .5555984761 .874593733 


\section{References}

(1) Wang, J.; Wolf, R. M.; Caldwell, J. W.; Kollman, P. A.; Case, D. A. Development and testing of a general amber force field. Journal of Computational Chemistry 2004, 25, 1157-1174. 